\title{
Actividad supresora del millerenólido sobre células mononucleares de sangre periférica humana
}

\author{
O mar A D upuy $L^{1,3 a}$, Renato Murillo2b, José A Bonilla $V^{1, b}$. \\ Millerenolide induces suppressive \\ activity on human peripheral blood \\ mononuclear cells
}

Background: Natural products are used in the production of therapeutic drugs due to their wide diversity and excellent adaptability to biological structures. Sesquiterpene lactones are the active constituents of several plants from the Asteraceae family. Aim: To assess the in vitro effect of a sesquiterpene lactone (millerenolide). Material and methods: The drug effect was assessed measuring the proliferation of lymphocytes using the 2,3bis (2-methoxy-4-nitro-5-sulfophenyl)-5-[(phenylamino) carbonyl]-2H-tetrazolium hydroxide (XTT) technique. Changes on the cell cycle were analyzed on a FACSort flow cytometer. The effect of millerenolide on the production of nitric oxide (NO) by macrophages was evaluated using the Griess reagent. Additionally, phagocytosis of latex particles and nitroblue tetrazolium (NBT) reduction by macrophages were evaluated microscopically. Results: Treatment of human peripheral blood mononuclear cells (PBMC) with millerenolide decreases the proliferation of lymphocytes, decreases the percentage of cells in S, and G2/M phases, and increases the proportion of cells in G0/G1 phase. Treatment of macrophages with millerenolide, reduces the production of NO, the phagocytic capacity and the number of cells able to reduce NBT. Cytotoxic effects of the lactone on human PBMC were only observed when the concentration was increased to $6 \mu \mathrm{g} / \mathrm{ml}$. Conclusions: Millerenolide could be considered as a potential therapeutic agent with immunosuppressive properties (Rev Méd Chile 2007; 135: 64-72).

(Key words: Immunosuppression; Lymphocytes; Sesquiterpenes)

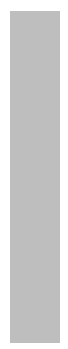

Recibido el 12 de septiembre, 2006. Aceptado el 4 de junio, 2007.

Investigación financiada, en parte, por el Servicio Alemán de Intercambio Académico (DAAD) y la Organización de las Naciones Unidas para la Educación, la Ciencia y la Cultura (UNESCO). Ninguna de las dos instituciones tuvo influencia en el diseño del estudio; ni en la recolección, análisis o interpretación de los datos; ni en la preparación, revisión o aprobación del manuscrito. ${ }^{1}$ Centro para Investigaciones en Biología Celular y Molecular (CIBCM). ${ }^{2}$ Escuela de Química. Universidad de Costa Rica, San José, Costa Rica. ${ }^{3}$ Laboratorio de Fisiología Animal e Inmunobiología Dr. Erich Graetz. Universidad de Panamá, Panamá, Panamá.

a MSc, candidato al grado de PhD en la Universidad de Costa Rica.

b $\mathrm{PhD}$

Correspondencia a: José A. Bonilla V. Centro de Investigación en Biología Celular y Molecular (CIBCM), Universidad de Costa Rica, San José, Costa Rica. Fax: 00506-207-3190. E mail: jbonilla@cariari.ucr.ac.cr 
$\mathrm{L}$ os productos naturales juegan un papel importante en el descubrimiento y elaboración de drogas como el paclitaxel (antineoplásico) y el simvastatin (antilipidémico) ${ }^{1}$.

Las lactonas sesquiterpénicas asociadas a principios activos, son aisladas de plantas como Parthenium hysterophorus ${ }^{2,3}$, Inula helenium, Arnica montana ${ }^{4}$ y Milleria quinqueflora ${ }^{5}$. Estos compuestos poseen un éster cíclico (lactona) que forma parte de una estructura central de 15 átomos de carbono y se caracterizan por tener un grupo $\alpha, \beta$ insaturado en el anillo lactónico, al que se atribuye gran parte de su actividad química ${ }^{4,6}$. El millerenólido es una lactona sesquiterpénica del grupo de los germacranólidos que posee un sistema anillado de 10 miembros con una gran flexibilidad conformacional ${ }^{7}$. Además, es bifuncional pues posee dos elementos estructurales $\alpha$, $\beta$ insaturados carbonil, que pueden inducir una fuerte actividad antiinflamatoria ${ }^{8}$. Algunas lactonas pueden actuar como agentes inmunorreguladores con espectro y actividad parecida a la ciclosporina y a los esteroides. Inhiben la interleucina 4 (IL-4) e IL-5, reducen la cantidad de células T, el infiltrado eosinófilo y la liberación de IL-3, interferón gama (IFN- $\gamma$ ), factor de necrosis tumoral alfa (TNF- $\alpha$ ) y el factor estimulante de colonias de granulocitos (G-CSF) 9,10 . También se han descrito propiedades antiinflamatorias, inhibición de la activación de linfocitos $\mathrm{T}$ y elevación de IL-2 en sangre total ${ }^{4,6}$.

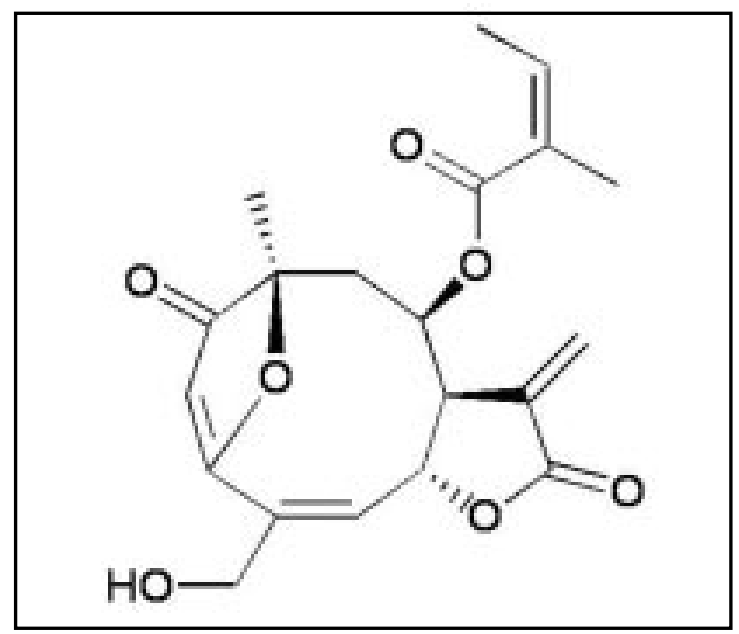

Figura 1. Estructura química del millerenólido.
Este estudio se realizó para evaluar el efecto in vitro del millerenólido (Figura 1) aislado de Viguiera sylvatica, sobre la producción de óxido nítrico (NO) y función fagocítica del macrófago, así como la proliferación y el ciclo celular de células mononucleares de sangre periférica (CMSP) humana. Nuestros resultados indican que, in vitro, el millerenólido causa la disminución de la producción de NO y de la capacidad fagocítica de los macrófagos. Además, provoca la disminución de la proliferación de linfocitos, del porcentaje de células en fase $S$ y $G_{2} / \mathrm{M}$ y el aumento del porcentaje de células en fase $G_{0} / G_{1}$.

\section{MATERIAL Y MÉTODO}

Millerenólido. El millerenólido fue aislado y purificado de V sylvatica según lo describen Castro et al ${ }^{5}$. Se preparó una solución $1 \mathrm{mg} / \mathrm{ml}$ ("stock") en RPMI 1640 y 10\% dimetilsulfóxido (DMSO) como solvente.

Obtención de CMSP. Luego de aprobada la investigación por el Programa de Doctorado de la UCR y previa información y consentimiento de voluntarios aparentemente sanos, las muestras de sangre total fueron colectadas en tubos estériles al vacío, con heparina como anticoagulante. Las CMSP fueron separadas mediante un gradiente de Ficoll-Hypaque como lo indica el fabricante (Sigma). Brevemente, la sangre se diluyó 1:2 en tampón de fosfatos (PBS) y se colocó sobre un colchón de 2 $\mathrm{ml}$ Ficoll-Hypaque 1,044. Luego se centrifugó a $1.800 \mathrm{rpm}$ por $45 \mathrm{~min}$ a $20^{\circ} \mathrm{C}$. Se extrajeron las células mononucleares y se lavaron con PBS estéril, $\mathrm{pH}$ 7,2. Finalmente las células se resuspendieron en RPMI 1640, suplementado con suero fetal bovino (SFB) al 10\%, $50 \mu \mathrm{g} / \mathrm{ml}$ de estreptomicina, $100 \mathrm{UI} /$ $\mathrm{ml}$ de penicilina, $2 \mathrm{mM}$ de glutamina, $5 \mathrm{mM}$ de 2 mercaptoetanol, $10 \mathrm{mM}$ de solución MEM de aminoácidos esenciales, $45 \mathrm{mM}$ de bicarbonato de sodio, 0,8 mM de glucosa y $25 \mathrm{mM}$ de N-(2hidroexitietil) piperazina-N'-(ácido 2-etanosulfónico) (HEPES) como tampón. La viabilidad celular se verificó por observación microscópica utilizando la tinción vital azul Tripán y se ajustó la concentración a $1 \times 10^{6}$ células vivas $/ \mathrm{ml}$.

Viabilidad celular. Las CMSP tratadas y no tratadas con millerenólido (0-6 $\mu \mathrm{g} / \mathrm{ml})$ fueron incubadas a 
$37^{\circ} \mathrm{C}$, en una atmósfera al $5 \%$ de $\mathrm{CO}_{2}$. Luego de 72 h, la viabilidad de las células fue determinada con azul Tripán.

Ensayos de linfoproliferación. Se colocaron 1x105 células en $100 \mu \mathrm{l}$ de medio por pozo, en placas de 96 pozos fondo en "U» (Corning). Se agregó fitohemaglutinina-M (PHA-M, Sigma, No. Cat. L-2646) como mitógeno a concentración final de $10 \mu \mathrm{g} / \mathrm{ml}$. Además, se añadieron diferentes concentraciones de millerenólido $(0,0,5,1,0,1,5,2,0,2,5$ y $3,0 \mu \mathrm{g} / \mathrm{ml})$. El volumen final de cada pocillo $(200 \mu \mathrm{l})$ se ajustó con RPMI 1640. Se incluyeron los controles: sólo CMSP, CMSP+PHA-M, $\mathrm{CMSP}+$ millerenólido, CMSP+DMSO. Las placas fueron incubadas a $37^{\circ} \mathrm{C}$ y $5 \%$ de $\mathrm{CO}_{2}$ por $72 \mathrm{~h}$.

La determinación de proliferación se realizó utilizando el método descrito por Mosmann ${ }^{11}$ con las modificaciones hechas por Bonilla y cols ${ }^{12}$. Brevemente, se emplearon $50 \mu$ por pocillo de una solución fresca de XTT (Sigma, No. Cat. X-4251) (1 $\mathrm{mg} / \mathrm{ml})$ y $\mathrm{N}$-metilfenacina metasulfato (PMS) $(0,01$ $\mathrm{M})$. Las células fueron incubadas durante $2 \mathrm{~h}$ a $37^{\circ} \mathrm{C}$ en oscuridad y, seguidamente, se cuantificó el cambio de color en un lector de microplacas Dynex MRX (Dynex Technologies) a $450 \mathrm{~nm}$ y con un filtro de referencia de $650 \mathrm{~nm}$.

Los resultados son expresados como porcentaje de estimulación, en donde el 100\% de estimulación corresponde a CMSP con PHA-M sin millerenólido (control).

Citometría deflujo. Las diferentes fases del ciclo celular fueron determinadas por análisis de ADN en citometría de flujo ${ }^{13}$, utilizando las células de los ensayos de linfoproliferación descritos anteriormente. Las células de cada pocillo fueron extraídas, centrifugadas y lavadas una vez con PBS. El botón fue resuspendido y fijado con etanol (70\%), durante 15 min en frío. Luego las células fueron centrifugadas y resuspendidas en 1 $\mathrm{ml}$ de solución del fluorocromo yoduro de propidio (PI) $(0,05 \mathrm{mg} / \mathrm{ml}$ de PI; 0,02 mg/ml de RNasa; 0,3\% de Nonidet P-40; $1 \mathrm{mg} / \mathrm{ml}$ de citrato de sodio) y se incubaron durante $1 \mathrm{~h}$ a $4^{\circ} \mathrm{C}$. El porcentaje de células en cada fase del ciclo celular fue calculado usando un citómetro FACScalibur (Becton Dickinson Mountain View, CA) y el programa CellQuest Pro. El equipo fue programado para registrar $1 \times 10^{4}$ eventos.

Obtención de monocitos/macrófagos humanos. $200 \mu \mathrm{l}$ de la suspensión de CMSP (1×10 6 células) $\mathrm{ml}$ ), se incubaron en placas de 96 pocillos con fondo plano (Costar) durante toda la noche, para permitir la adhesión de los monocitos/macrófagos al fondo de los pocillos. Luego el sobrenadante con células no adherentes fue extraído, se realizaron lavados con medio RPMI 1640 y se restituyó el volumen a $200 \mu \mathrm{l}$ con medio de cultivo fresco.

Las células adherentes, fundamentalmente monocitos y macrófagos, fueron incubadas en cámara húmeda, a $37^{\circ} \mathrm{C}$ y $5 \%$ de $\mathrm{CO}_{2}$.

Producción de óxido nítrico por monocitos/macrófagos humanos. Monocitos/macrófagos tratados y no tratados con millerenólido $(0-3 \mu \mathrm{g} / \mathrm{ml})$, fueron activados con $50 \mu \mathrm{l}$ de lipopolisacárido (LPS) $(1 \mu \mathrm{g} / \mathrm{ml}$ en RPMI 1640) y se les incubó a $37^{\circ} \mathrm{C}$ durante $24 \mathrm{~h}$. Posteriormente, se transfirieron $100 \mu \mathrm{l}$ de sobrenadante de cada pocillo a otra placa y se agregó, igual volumen de reactivo de Griess (1\% de sulfanilamida, Sigma, No. Cat. S-9251 y 0,1\% de naftilenediamina, Sigma, No. Cat. N-9125 disuelto en ácido fosfórico al $5 \%$ ). Luego se determinó la absorbancia ( $\lambda 550 \mathrm{~nm}$ ) utilizando un lector de microplacas.

Reducción de nitroazul de tetrazolio (NBT). Monocitos/macrófagos tratados y no tratados con millerenólido $(0-2 \mu \mathrm{g} / \mathrm{ml})$, fueron estimulados con $10 \mu \mathrm{l}$ de LPS (Sigma, No. Cat. 840-15) $(1 \mu \mathrm{g} / \mathrm{ml})$ y $25 \mu \mathrm{l}$ de NBT (Sigma, No. Cat. 840-10) $(1 \mathrm{mg} / \mathrm{ml})$ y luego incubados a $37^{\circ} \mathrm{C}$ durante $20 \mathrm{~min}$. El sobrenadante fue descartado y las células fueron teñidas con Giemsa y observadas al microscopio para determinar la cantidad de macrófagos NBT positivos (células con depósitos azules). Se realizó lo mismo con macrófagos no estimulados.

Función fagocítica. Monocitos/macrófagos tratados y no tratados con millerenólido $(0-3 \mu \mathrm{g} / \mathrm{ml})$, fueron incubados a $37^{\circ} \mathrm{C}$ durante $24 \mathrm{~h}$ con la suspensión de partículas de látex teñidas de azul oscuro $(0,846 \mu \mathrm{m}$ de diámetro; Sigma, No. Cat. L-1398), en una relación macrófago:partículas $=1: 30$. Posteriormente, las células fueron lavadas con PBS frío para eliminar las partículas libres, se fijaron con metanol y se tiñeron con Giemsa. Las placas fueron observadas al microscopio para determinar el porcentaje de células con capacidad fagocítica y la cantidad promedio de partículas de látex fagocitadas por macrófago. 
Estadística. Los datos fueron evaluados por medio de un análisis de varianza (ANOVA) y la prueba de Dunnett. Todas las pruebas estadísticas fueron realizadas con el SigmaStat Statistical Analysis Software (Jandel Corporation). Los resultados se presentan como el promedio de cinco réplicas de cada tratamiento y de los controles \pm la desviación estándar respectiva. Un valor de $\mathrm{p}<0,05$ fue considerado como nivel de significancia estadística.

\section{Resultados}

Con el propósito de medir el efecto tóxico del millerenólido sobre las CMSP, se realizó un ensayo de incorporación de azul Tripán, que muestra que las células mantienen una viabilidad cercana a 90\% cuando son tratadas con concentraciones de millerenólido $\leq 6 \mu \mathrm{g} / \mathrm{ml}$ (Figura 2A). Las concentraciones usadas en todos nuestros experimentos son $\leq 3 \mu \mathrm{g} / \mathrm{ml}$.

Por otra parte, para estudiar el efecto del millerenólido sobre la capacidad proliferativa de las CMSP, se midió el porcentaje de estimulación de las CMSP en presencia y ausencia del millerenólido. Se pudo observar un efecto dependiente de la dosis que consistió en una disminución significativa $(\mathrm{p}<0,05)$ de la respuesta linfoproliferativa a PHA-M en los cultivos de CMSP tratados con el millerenólido, mientras que el porcentaje

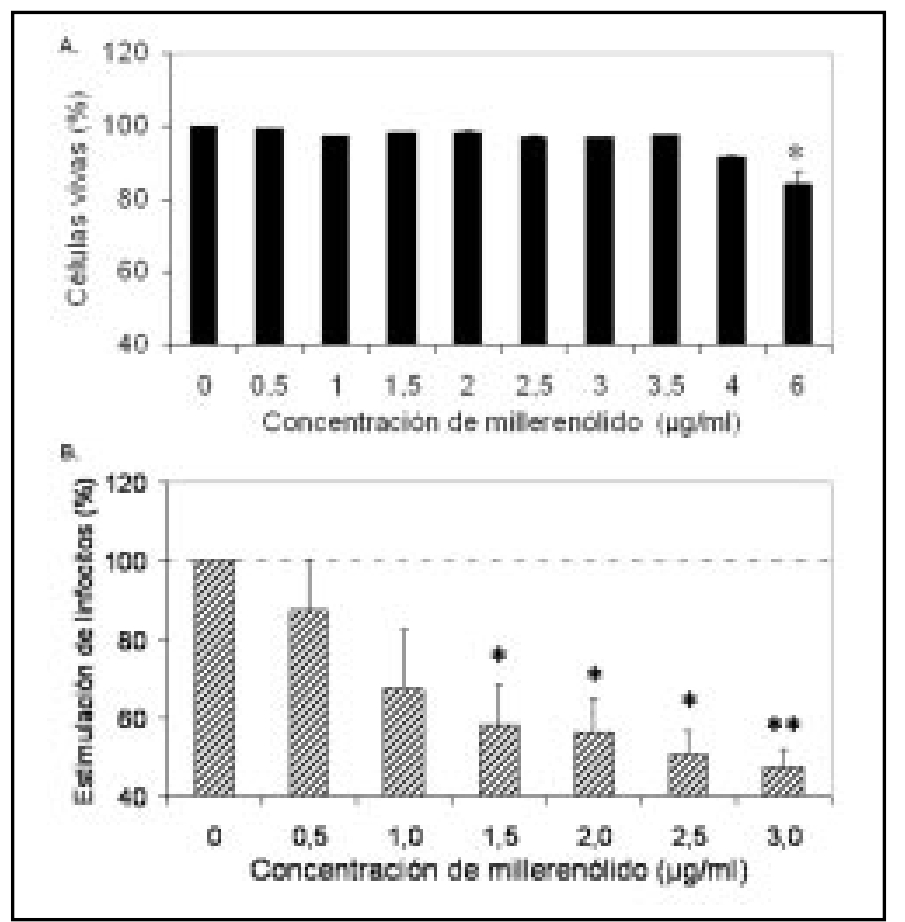

Figura 2. Efecto in vitro del millerenólido sobre CMSP. A. Análisis de toxicidad del millerenólido sobre CMSP. Las células fueron cultivadas en ausencia y en presencia de diferentes concentraciones de millerenólido ( 0 - 6,0 $\mathrm{mg} / \mathrm{ml}$ ). El número de células vivas y muertas fue determinado con azul tripán. El número de células viables disminuyó a partir de $6,0 \mu \mathrm{g} / \mathrm{ml}$ de millerenólido. B. Efecto in vitro del millerenólido sobre la respuesta linfoproliferativa. CMSP humanas fueron cultivadas con PHA-M $(10 \mu \mathrm{g} / \mathrm{ml})$ y diferentes concentraciones de millerenólido $(0-3,0 \mu \mathrm{g} / \mathrm{ml})$. Las células fueron incubadas con XTT y se midió la absorbancia del sobrenadante. Los resultados se expresan como porcentaje de estimulación. La proliferación de linfocitos disminuyó de una manera dependiente de la dosis en los cultivos tratados con el millerenólido. Cada columna representa el promedio $\pm \mathrm{DE}$ de cinco réplicas. ${ }^{*} \mathrm{y}$ ** Indican diferencia estadísticamente significativa $(\mathrm{p}<0,05$ y $\mathrm{p}<0,01$, respectivamente) con respecto al grupo control $100 \%$ de estimulación (sin millerenólido). 
de células viables se mantuvo en 100\% (Figura 2A, B). El efecto del millerenólido alcanzó su máxima a una concentración de $3 \mu \mathrm{g} / \mathrm{ml}$ cuando la disminución de la proliferación celular fue cercana a 50\% (Figura 2B).

Para determinar el efecto del millerenólido sobre la progresión de las CMSP a lo largo del ciclo celular, las células estudiadas fueron teñidas con PI y analizadas con citometría de flujo después de los ensayos de proliferación. Se observó que el porcentaje de CMSP en fase $\mathrm{S}$ y $\mathrm{G}_{2} / \mathrm{M}$ disminuyó después del tratamiento con el millerenólido $(3 \mu \mathrm{g} / \mathrm{ml})$. Además, hubo un aumen-

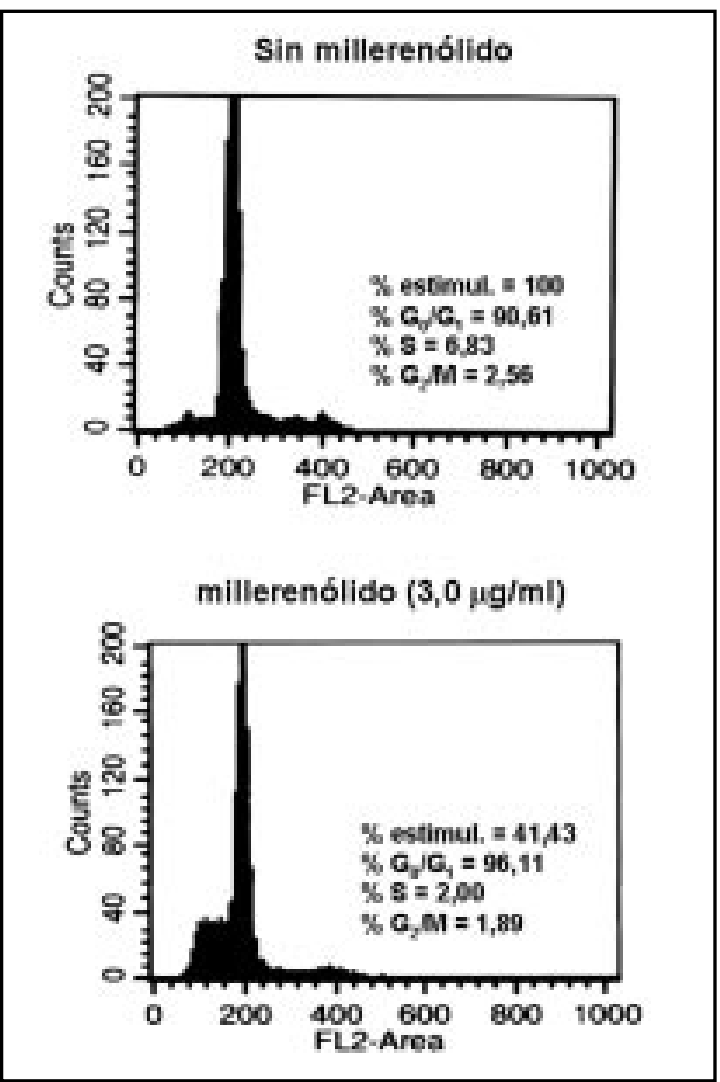

Figura 3. Citometría de flujo de las CMSP. Después de los ensayos de linfoproliferación, las células fueron teñidas con PI y analizadas con FACS. El millerenólido $(3 \mu \mathrm{g} / \mathrm{ml})$ causó la disminución significativa del porcentaje de células en fase $S$ y $G_{2} / \mathrm{M}$ y el aumento del porcentaje de células en fase $G_{0} / G_{1}$ con respecto al control estimulado (sin millerenólido). tó en el porcentaje de células en fase $G_{0} / G_{1}$ en dichos cultivos (Figura 3). Estos resultados concuerdan con la disminución en la proliferación celular observada durante los ensayos de proliferación (Figura 2B y 3).

Para evaluar la capacidad antioxidante del millerenólido sobre los macrófagos, se realizó un ensayo con el reactivo de Griess y el ensayo de reducción de NBT. Se pudo observar que los macrófagos tratados con $2 \mu \mathrm{g} / \mathrm{ml}$ de millerenólido, disminuyeron significativamente la producción de NO (Figura 4) y la capacidad de reducir NBT (Figura 5).

Con el propósito de evaluar el efecto del millerenólido sobre la fagocitosis, se realizó un ensayo consistente en cuantificar la cantidad de partículas de látex fagocitadas por los macrófagos cultivados en presencia y ausencia del millerenólido. Este ensayo mostró que los macrófagos tratados con millerenólido $(2 \mu \mathrm{g} / \mathrm{ml})$ fagocitan menos partículas que aquellos que no fueron tratados con el millerenólido (Figura 6). No se observaron diferencias entre tratamientos y controles en cuanto al número de células fagocíticas viables.

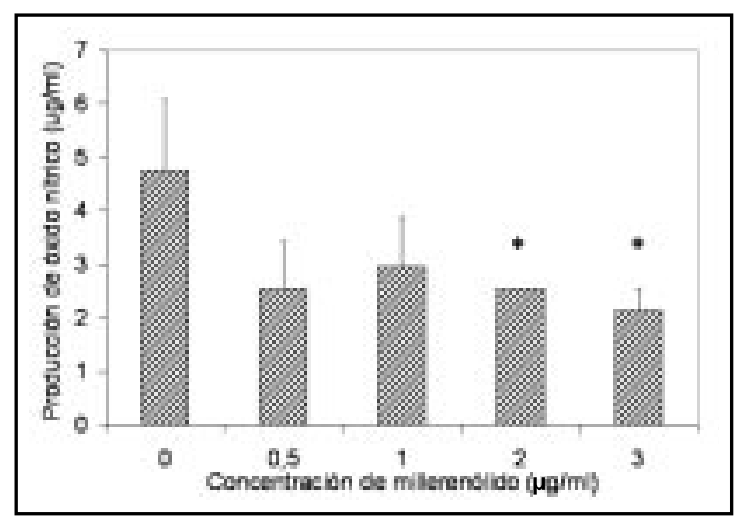

Figura 4. Efecto del millerenólido sobre la producción in vitro de óxido nítrico por macrófagos activados. Las células fueron cultivadas con LPS, en presencia y ausencia de millerenólido $(0-3 \mu \mathrm{g} / \mathrm{ml})$, y se midió la concentración de NO del sobrenadante. La producción de óxido nítrico disminuyó en presencia del millerenólido $(2 \mu \mathrm{g} / \mathrm{ml})$. Cada columna representa el promedio \pm DE de cinco réplicas. * Indica diferencia estadísticamente significativa $(\mathrm{p}<0,05)$ con respecto al grupo control (sin millerenólido). 


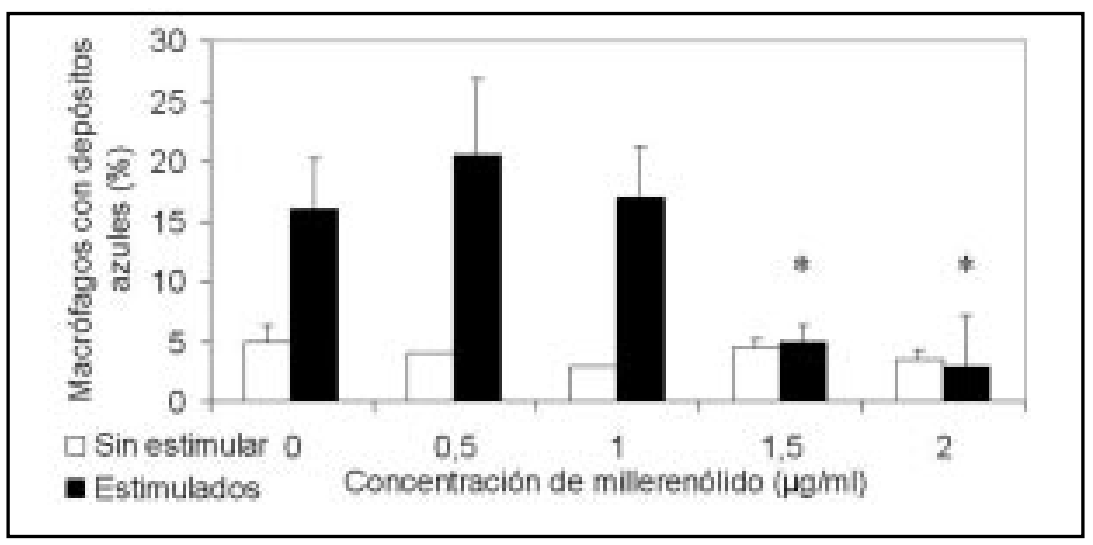

Figura 5. Efecto del millerenólido sobre la reducción de NBT por macrófagos. Células tratadas y no tratadas con diferentes concentraciones de millerenólido $(0-2 \mu \mathrm{g} / \mathrm{ml})$ fueron cultivadas con y sin LPS y luego fueron incubadas con NBT. Los macrófagos NBT positivos (células con depósitos azules) fueron contabilizados. La cantidad de macrófagos capaces de reducir NBT disminuyó después del tratamiento con el millerenólido ( $\geq 1,5$ $\mu \mathrm{g} / \mathrm{ml})$. Cada columna representa el promedio \pm DE de cinco réplicas. *Indica diferencia estadísticamente significativa $(\mathrm{p}<0,05)$ entre tratamiento y el grupo control estimulado (sin millerenólido).

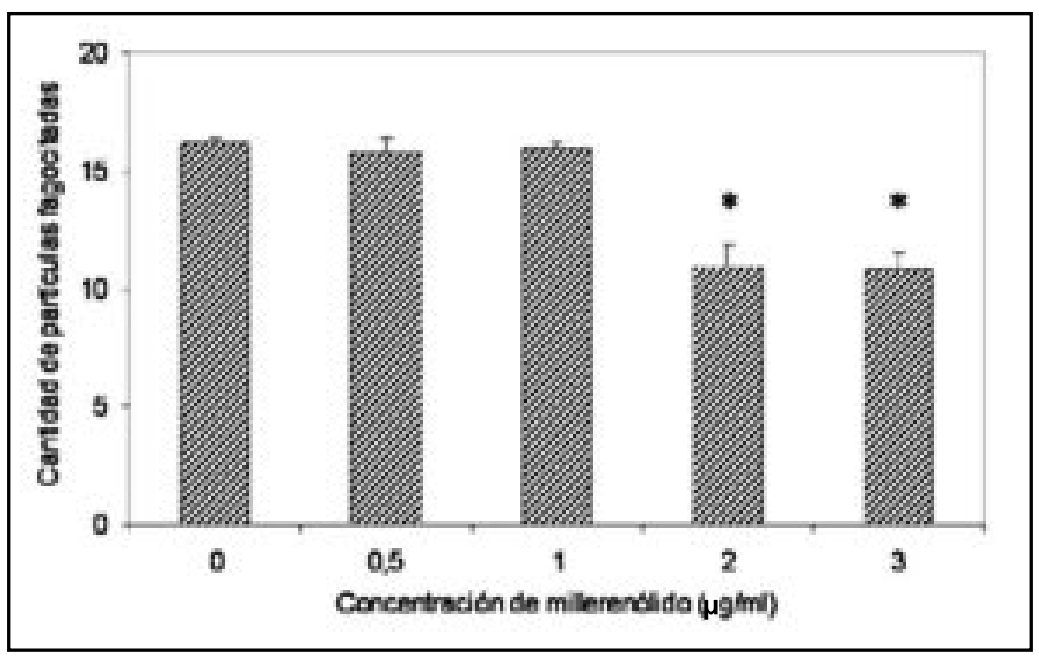

Figura 6. Efecto in vitro del millerenólido sobre la fagocitosis. Macrófagos tratados y no tratados con diferentes concentraciones de millerenólido $(0-3 \mu \mathrm{g} / \mathrm{ml})$ fueron incubados con partículas de látex y se contabilizó el número de partículas fagocitadas por macrófago. La cantidad de partículas fagocitadas por los macrófagos disminuyó en los cultivos tratados con el millerenólido $(2 \mu \mathrm{g} / \mathrm{ml})$. Cada columna representa el promedio \pm DE de cinco réplicas. * Indica diferencia estadísticamente significativa $(\mathrm{p}<0,05)$ entre tratamiento y el grupo control (sin millerenólido).

DISCUSIÓN

El millerenólido interfiere con la respuesta proliferativa de las CMSP, deteniendo su ciclo celular en la fase $G_{0} / G_{1}$ (Figuras 2 y 3). La actividad supresora del millerenólido sobre la linfoproliferación es dosis dependiente y no puede atribuirse a muerte celular, ya que se determinó que el 
millerenólido no es citotóxico in vitro a concentraciones $<6 \mu \mathrm{g} / \mathrm{ml}$ (Figura 2A, B).

El efecto supresor del millerenólido sobre la respuesta de los linfocitos al mitógeno (Figura 2), es consistente con lo reportado por varios investigadores, algunas lactonas sesquiterpénicas inhiben selectivamente la unión del factor nuclear $\mathrm{\kappa B}$ (NF-KB) al $\mathrm{ADN}^{5,8,14,15} \mathrm{y}$, en consecuencia, inhiben la liberación de IL-1ß, TNF- $\alpha$, IL- 6 , IL-8 ${ }^{16,17}$, IL-2, IL-4 e IFN- ${ }^{18}$.

El NF- $\mathrm{KB}$ es necesario para promover la activación y función efectora de las células $\mathrm{T}$ y $\mathrm{B}$, macrófagos y otras ${ }^{19}$.

Este factor de transcripción regula múltiples genes que participan en la producción de diferentes citocinas involucradas en diferentes eventos de la respuesta inmune ${ }^{20,21}$. De allí que, su inhibición farmacológica podría ser de utilidad en la modulación de la inflamación ${ }^{22}$ y de otras funciones del sistema inmune. La evidencia indica que el mecanismo general de acción de las lactonas sesquiterpénicas, es por interacción directa con NF- $\mathrm{KB}^{6}$. La alquilación selectiva de grupos sulfihidrilos de los residuos de cisteínas en la subunidad p65 de NF$\kappa \mathrm{B}$, evita su unión al $\mathrm{ADN}^{6,14}$. Esto es posible, debido a que las lactonas sesquiterpénicas poseen grupos $\alpha, \beta$ ó $\alpha, \beta, \gamma$ carbonil insaturados, tales como $\alpha$-metilen- $\beta$-lactonas ó $\alpha$, $\beta$-ciclopentanonas no sustituidas. Estos grupos funcionales reaccionan con grupos nucleofílicos, especialmente con los sulfihidrilos de las cisteínas presentes en NF- $\kappa B$, en una adición tipo Michael ${ }^{23,24}$.

Los macrófagos son células versátiles que toman parte en la respuesta inmune inespecífica contra diferentes microorganismos ${ }^{25}$. Una vez activados, pueden generar grandes cantidades de NO a partir de L-arginina por acción de la sintasa de NO inducible (iNOS, del inglés inducible nitric oxide synthase) ${ }^{26,27}$. El NO es un mediador celular de múltiples funciones biológicas ${ }^{28}$, incluyendo citotoxicidad mediada por macrófagos, destrucción de partículas fagocitadas, neurotransmisión y relajación del músculo liso ${ }^{29}$.

Paradójicamente, la actividad de iNOS, inducida por LPS e IFN- $\gamma$, correlaciona inversamente con la vida media de los macrófagos en cultivo ${ }^{30}$. Los radicales libres y citocinas proinflamatorias están implicados en la patogénesis del "shock" endotóxico, una patología con alta mortalidad causada por endotoxinas bacterianas gram-negativas. Es posible que terapias con antioxidantes y captadores de radicales libres reduzcan los efectos negativos de este proceso ${ }^{31}$. En contraste al efecto observado sobre la linfoproliferación, la actividad supresora del millerenólido sobre los macrófagos no es dosis dependiente, lo que sugiere que el millerenólido actúa a través de diferentes mecanismos.

El número de macrófagos capaces de reducir NBT aumenta por la estimulación con LPS. De esta forma, el efecto del millerenólido pudo ser estudiado en una población de macrófagos expuesta y no expuesta a LPS con el fin de determinar si esta lactona puede disminuir la producción de especies reactivas del oxígeno (ROS, del inglés "reactive oxygen species"), ya que éstos reducen el NBT en respuesta a la estimulación con LPS. En este sentido, el millerenólido causó la disminución del número de macrófagos capaces de reducir NBT en aquellos cultivos estimulados con LPS pero no en los cultivos que no fueron estimulados. Esta disminución puede atribuirse a un efecto supresor del millerenólido sobre la producción de ROS inducida y no sobre la poca producción basal o constitutiva de los macrófagos cultivados (Figuras 4 y 5).

Nuestros hallazgos resultan de interés, si consideramos que la producción de ROS, necesaria para destruir partículas o microorganismos fagocitados, sólo resulta beneficiosa si sus niveles son regulados adecuadamente. De hecho, si la producción de ROS, excede la capacidad defensiva antioxidante de las células y fluidos extracelulares, provoca daños oxidativos en muchos órganos, como sucede durante un "shock" endotóxico o falla orgánica múltiple ${ }^{31}$. Sustancias como el millerenólido, pueden ser útiles para modular la activación de los macrófagos.

El hecho de que la disminución en la producción de NO (Figuras 4 y 5), se acompañara de una disminución en la capacidad fagocítica de los macrófagos (Figura 6), indica que este millerenólido interfiere con la fagocitosis in vitro, afectando vías que podrían no ser compartidas con las utilizadas en la síntesis de NO. Se ha observado que otras lactonas sesquiterpénicas influyan sobre diferentes procesos celulares involucrados en el inicio y progreso de la inflamación ${ }^{4}$. 
El estrés oxidativo ligado a la producción de radicales libres y citocinas proinflamatorias, puede afectar otras funciones de los macrófagos, como la adherencia a tejidos, liberación de factores quimiotácticos y la misma capacidad de fagocitar ${ }^{31}$. Esto sugiere que agentes antioxidantes como el millerenólido acá estudiado, podrían ser útiles para regular las funciones del macrófago en situaciones de estrés oxidativo.

En resumen, este millerenólido, utilizado in vitro en concentraciones no tóxicas, es capaz de disminuir la linfoproliferación, la producción de NO y la capacidad fagocítica de los macrófagos, por lo que puede ser considerado como un agente con potencial actividad inmunomoduladora para futuros estudios in vivo.

\section{REFERENCIAS}

1. Butler MS. The Role of Natural Product Chemistry in Drug Discovery. J Nat Prod 2004; 67: 2141-53.

2. Mew D, Balza F, Towers GHN, Ley JG. Antitumor effects of the sesquiterpene lactone parthenir. Planta med 1982; 45: 23-7.

3. Águila B, Meneses R, González L, Madrigal E, FERnÁndez D. Extracto acuso de escoba amarga. Estudio preliminar de sus propiedades. Rev $\mathrm{Cu}-$ bana Plant Med 2000; 5: 123-4.

4. Humar M, García-Piñeres AJ, Castro V, Merfort I. Effect of sesquiterpene lactones on the expression of the activation marker CD69 and of IL-2 in T-lymphocytes in whole blood. Biochem Pharmacol 2003; 65: 1551-63.

5. Castro V, Rüngeler P, Murillo R, Hernandez E, Mora G, PAHL HL eT al. Study of sesquiterpene lactones from Milleria quinqueflora on their anti-inflammatory activity using the transcription factor NFkappa B as molecular target. Phytochemistry 2000; 53: 257-63.

6. García-Piñeres AJ, Castro V, Mora G, Schmidt TJ, Strunck E, Pahl HL et al. Cysteine 38 in p65/NFkkkB plays a crucial role in DNA binding inhibition by sesquiterpene lactones. J Biol Chem 2001; 276(43): 39713-20.

7. Siedle B, Gustavsson L, Johansson S, Murillo R, Castro V, Bohlin L et al. The effect of sesquiterpene lactones on the release of human neutrophil elastase. Biochem Pharmacol 2003; 65: 897-903.

\section{Agradecimientos}

Agradecemos al Servicio Alemán de Intercambio Académico (DAAD) y a la Organización de las Naciones Unidas para la Educación, la Ciencia y la Cultura (UNESCO), por financiar, en parte, esta investigación. Esta publicación es uno de los requisitos del Programa de Doctorado en Ciencias de la Universidad de Costa Rica (UCR), para otorgar el grado de Doctor en Ciencias a Omar A. Dupuy Loo.

Se agradece a la Dra. Berta Valverde, del Hospital Nacional de Niños (Costa Rica) y Rodrigo Mora, de la Facultad de Microbiología de la UCR, por la colaboración brindada para la realización de los análisis con el citómetro de flujo.

8. Rüngeler P, Castro V, Mora G, Gören N, VischnewsKI W, PAHL HL ET AL. Inhibition of transcription factor NF- $\mathrm{KB}$ by sesquiterpene lactones: a proposed molecular mechanism of action. Bioorg Med Chem 1999; 7: 2343-52.

9. Donald YM. Pathogenesis of atopic dermatitis. Clin Immunol 1999; 104: s99-1089.

10. Fleischer A. Treatment of atopic dermatitis: role of tracolimus ointment as a topical noncorticosteroidal therapy. J Allergy Clin Immunol 1999; 104: S126-130.

11. Mosmann T. Rapid colorimetric assay for cellular growth and survival: application to proliferation and cytotoxicity assays. J Immunol Methods 1983; 65: 55-63.

12. Bonilla Ja, Mesén MG, Cartín W. Modificación de un método colorimétrico que usa XTT, para la determinación de linfoproliferación. Rev Cost Cienc Med 1993; 14(1,2): 25-31.

13. Cortés-Bratti X, Karlsson C, Lagergard T, ThelesTAM M, Frisan T. The Haemophilus ducreyi cytolethal distending toxin induces cell cycle arrest and apoptosis via the DNA damage checkpoint pathways. J Biol Chem 2001; 276: 5296-302.

14. Ly G, Knorre A, Schmidt TJ, Pahl HL, Merfort I. The anti-inflammatory sesquiterpene lactone helenalin inhibits the transcription factor NF- $\mathrm{BB}$ by directly targeting p65. J Biol Chem 1998; 273: 33508-16.

15. Wong HR, MenÉndez IY. Sesquiterpene lactones inhibit inducible nitric oxide synthase gene ex- 
pression in cultured rat aortic smooth muscle cells. Biochem Biophys Res Cummun 1999; 262: 375-80.

16. Cavallini L, Francesconi Ma, Zoccarato F, Alexandre A. Involvement of nuclear factor-kappa B (NF$\kappa \mathrm{B})$ activation in mitogen-induced lymphocyte proliferation: inhibitory effects of lymphoproliferation by salicylates acting as NF- $\mathrm{KB}$ inhibitors. Biochem Pharmacol 2001; 62: 141-7.

17. Koch E, Klaas CA, Rüngeler P, Castro V, Mora G, VISCHNEWSKI W ET AL. Inhibition of inflammatory cytokine production and lymphocyte proliferation by structurally different sesquiterpene lactones correlates with their effect on activation of NF-кB. Biochem Pharmacol 2001; 62: 795-801.

18. Li-Weber M, Giaisi M, Treiber MK, Krammer PH. The anti-inflammatory sesquiterpene lactone parthenolide suppresses IL-4 gene expression in peripheral blood T. Eur. J. Immunol. 2002; 32: 3587-97.

19. Ruland J, MaK TW. Transducing signals from antigen receptors to nuclear factor $\kappa \mathrm{B}$. Immunological Reviews 2003; 193: 93-100.

20. LeE JI, Burckart GJ. Nuclear factor kappa B: important transcription factor and therapeutic target. J Clin Pharmacol 1998; 38: 981-93.

21. KuKLina EM, SHIRShev SV. Role of transcription factor NFAT in the immune response. Biochemistry (Mosc) 2001; 66: 467-75.

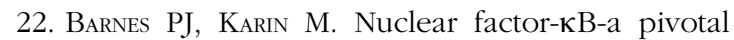
transcription factor in chronic inflammatory diseases. N Engl J Med 1997; 336: 1066-71.

23. Dupus G, Mitchell JC, Towers GHN. Reaction of alantolactone, an allergenic sesquiterpene lactone, with some amino acids. Resultant loss of immunologic reactivity. Can J Biochem 1974; 52: 575-81.

24. Picman AK, Rodríguez E, Towers GHN. Formation of adducts of parthenin and related sesquiterpene lactones with cysteine and glutathione. Chem Biol Interact 1979; 28: 83-9.

25. Hirvonen MR, Brüne B, Lapetina EG. Heat shock proteins and macrophage resistance to the toxic effects of nitric oxide. Biochem J 1996; 315: 845-849.

26. Stuehr DJ, Marletta MA. Mammalian nitrate biosynthesis: mouse macrophages produce nitrite and nitrate in response to Escherichia coli lipopolysaccharide. Proc Natl Acad Sci USA 1985; 82: 7738-42.

27. Hibbs JB, Taintor RR, Vavrin Z, Rachlin EM. Nitric oxide: a cytotoxic activated macrophage effector molecule. Biochem Biophys Res Commun 1988; 157: 87-94.

28. NATHAN C. Nitric oxide as a secretory product of mammalian cells. FASEB J 1992; 6: 3051-3064.

29. Hibbs JB, Vavrin Z, Taintor RR. L-arginine is required for expression of the activated macrophage effector mechanism causing selective metabolic inhibition in target cells. J Immunol 1987; 138: 550-65.

30. Albina JE, Mills CD, Henry Wl, Caldwell MD. Regulation of macrophage physiology by Larginine: role of the oxidative L-arginine deiminase pathway. J Immunol 1989; 143: 3641-6.

31. Víctor VM, De la Fuente $M$. Changes in the superoxide production and other macrophage functions could be related to the mortality of mice with endotoxin-induced oxidative stress. Physiol Res 2003; 52: 101-10. 